\title{
CORRECTION
}

\section{Correction to: Trends in clinical profiles, organ support use and outcomes of patients with cancer requiring unplanned ICU admission: a multicenter cohort study}

Fernando G. Zampieri ${ }^{1,2,3}$, Thiago G. Romano ${ }^{4,5,9}$, Jorge I. F. Salluh ${ }^{6}$, Leandro U. Taniguchi ${ }^{7,8}$, Pedro V. Mendes ${ }^{4,8,9}$, Antonio P. Nassar Jr ${ }^{10}$, Roberto Costa ${ }^{11}$, William N. Viana ${ }^{12}$, Marcelo O. Maia ${ }^{13,14}$, Mariza F. A. Lima ${ }^{15}$, Sylas B. Cappi ${ }^{16}$, Alexandre G. R. Carvalho ${ }^{17}$, Fernando V. C. De Marco ${ }^{18}$, Marcelo S. Santino ${ }^{19}$, Eric Perecmanis ${ }^{20}$, Fabio G. Miranda ${ }^{21}$, Grazielle V. Ramos ${ }^{6}$, Aline R. Silva ${ }^{6}$, Paulo M. Hoff ${ }^{1,22}$, Fernando A. Bozza ${ }^{6,23}$ and Marcio Soares ${ }^{6 *}$ (1)

(c) 2020 Springer-Verlag GmbH Germany, part of Springer Nature

\section{Correction to: Intensive Care Med} https://doi.org/10.1007/s00134-020-06184-2

The original version of this article unfortunately contained a mistake in Fig. 4. The corrected figure (Fig. 4) can be found below. The original version was updated. We apologize for the mistake. 


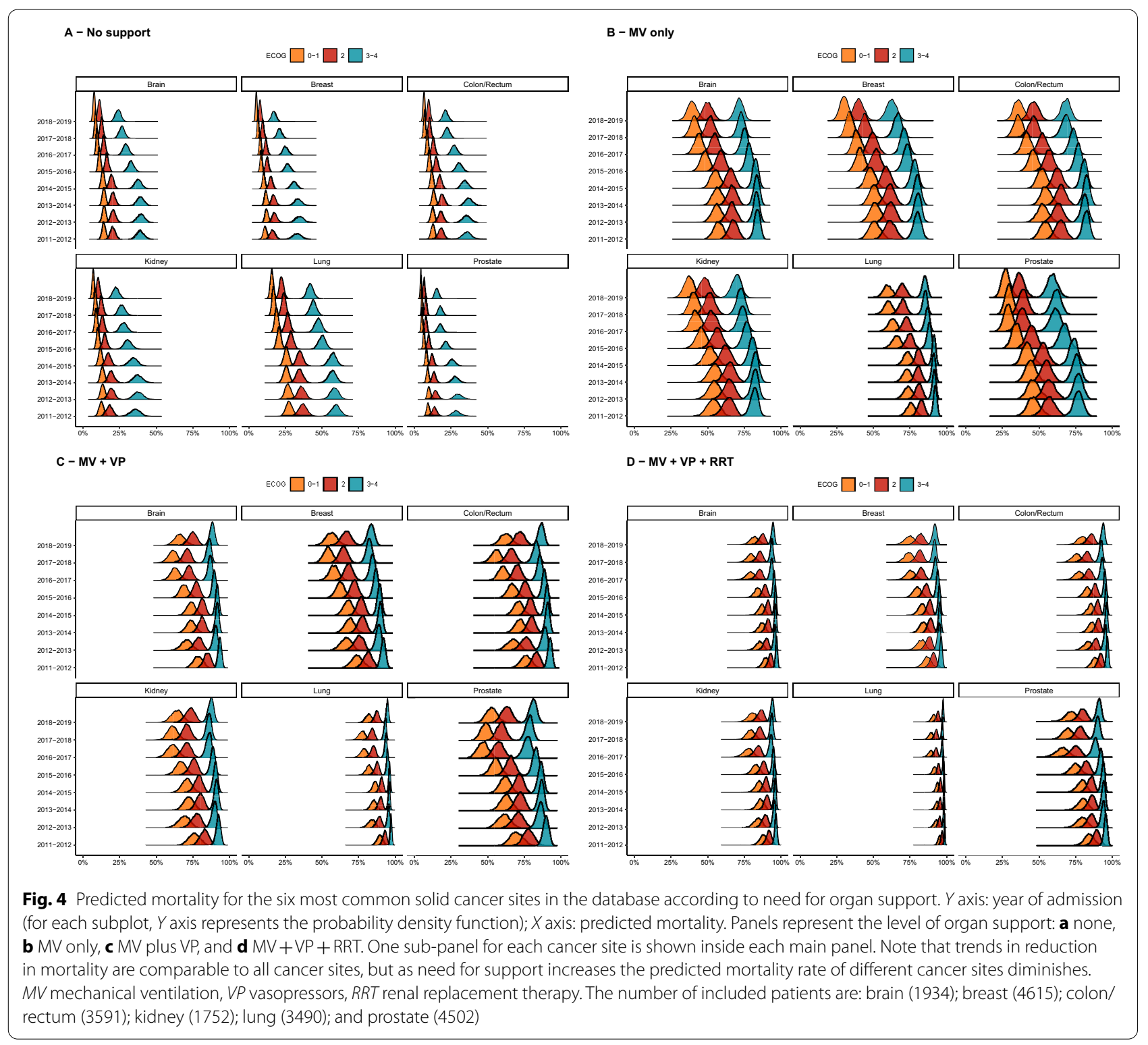

\section{Author details}

${ }^{1}$ Department of Critical Care, D'Or Institute for Research and Education, 30. Botafogo, Rio de Janeiro, Brazil. ${ }^{2}$ Research Institute, HCor, São Paulo, Brazil. ${ }^{3}$ Center of Epidemiological and Clinical Research, Southern Denmark University, Odense, Denmark. ${ }^{4}$ Intensive Care Unit, Hospital Vila Nova Star, São Paulo, Brazil. ${ }^{5}$ Nephrology Department, ABC Medical School, Santo André, Brazil. ${ }^{6}$ Department of Critical Care and Graduate Program in Translational Medicine, D'Or Institute for Research and Education, Rio de Janeiro, Brazil. ${ }^{7}$ Research and Education Institute, Hospital Sírio-Libanês, São Paulo, Brazil. ${ }^{8}$ Emergency Medicine Discipline, University of São Paulo, São Paulo, Brazil. ${ }^{9}$ Oncological Intensive Care Unit, Unidade Itaim, Hospital São Luiz, São Paulo, Brazil. ${ }^{10}$ Intensive Care Unit, A.C. Camargo Cancer Center, São Paulo, Brazil. ${ }^{11}$ Intensive Care Unit, Hospital Quinta D'Or, Rio de Janeiro, Brazil. ${ }^{12}$ Intensive Care Unit, Hospital Copa D'Or, Rio de Janeiro, Brazil. ${ }^{13}$ Intensive Care Unit, Hospital Santa Luzia Rede D'Or São Luiz, Brasília, Brazil. ${ }^{14}$ Intensive Care Unit, Hospital DF Star Rede D'Or São Luiz, Brasília, Brazil. ${ }^{15}$ Intensive Care Unit, Hospital Esperança Recife, Recife, Brazil. ${ }^{16}$ Intensive Care Unit, Unidade Brasil, Hospital São Luiz, Santo
André, Brazil. ${ }^{17}$ Intensive Care Unit, UDI Hospital, São Luís, Brazil. ${ }^{18}$ Intensive Care Unit, Hospital ViValle, São José dos Campos, Brazil. ${ }^{19}$ Intensive Care Unit, Hospital Barra D'Or, Rio de Janeiro, Brazil. ${ }^{20}$ Intensive Care Unit, Hospital Caxias D'Or, Duque de Caxias, Brazil. ${ }^{21}$ Intensive Care Unit, Hospital Copa Star, Rio de Janeiro, Brazil. ${ }^{22}$ Oncologia D'Or, São Paulo, Brazil. ${ }^{23}$ Instituto Nacional de Infectologia Evandro Chagas, Fundação Oswaldo Cruz, Rio de Janeiro, Brazil.

\section{Publisher's Note}

Springer Nature remains neutral with regard to jurisdictional claims in published maps and institutional affiliations.

Published online: 2 October 2020 